\title{
On the Interplay of Magnetic and Molecular Forces in Curie-Weiss Ferrofluid Models
}

\author{
Hans-Otto Georgii ${ }^{1}$ and Valentin Zagrebnov ${ }^{2}$
}

Received February 8, 1998

\begin{abstract}
We consider a mean-field continuum model of classical particles in $\mathbf{R}^{d}$ with Ising or Heisenberg spins. The interaction has two ingredients, a ferromagnetic spin coupling and a spin-independent molecular force. We show that a feedback between these forces gives rise to a first-order phase transition with simultaneous jumps of particle density and magnetization per particle, either at the threshold of ferromagnetic order or within the ferromagnetic region. If the direct particle interaction alone already implies a phase transition, then the additional spin coupling leads to an even richer phase diagram containing triple (or higher order) points.
\end{abstract}

KEY WORDS: Classical continuous system; first-order phase transition; mean-field; tricritical; large deviations; maximum entropy principle.

\section{INTRODUCTION}

Classical systems of particles located in $\mathbf{R}^{d}$ and having some internal degrees of freedom are a natural object of physical study. Examples of such systems are

- ferromagnetic fluids, where each particle has an Ising or Heisenberg spin;

- liquid crystals consisting of long molecules with a dipole-dipole interaction;

- Coulomb gases of charged particles; and, more generally,

\footnotetext{
${ }^{1}$ Mathematisches Institut der Universität München, D-80333 Munich, Germany; e-mail: georgii $(a$ rz.mathematik.uni-muenchen.de.

${ }^{2}$ Département de Physique, Université de la Méditerranée, C.P.T., CNRS-Luminy-Case 907, F-13288 Marseille, Cedex 09, France; e-mail: zagrebnov(acpt.univ-mrs.fr.
} 
- multitype particle systems - the internal degrees of freedom then correspond to the types of the particles.

The last class includes the Widom-Rowlinson model of two types of particles with a hard-core interspecies repulsion, the first continuum model for which a phase transition was established rigorously. ${ }^{(29,22)}$ In a closely related ferrofluid model of the first class, spontaneous magnetization was established by Gruber and Griffiths. ${ }^{(12)}$ A common generalization of these models is the continuum Potts model, for which the existence of a phase transition was recently proved; ${ }^{(9)}$ see also the references therein for related work.

In all these examples, the phase transition originates from an interaction between the internal degrees of freedom, e.g., the spin orientations, and it manifests itself as an orientational order resembling the familiar situation in lattice spin models. ${ }^{(8,23)}$ For continuum models, however, one is primarily interested in a different kind of critical phenomenon, namely positional order, which corresponds to a liquid-vapor transition and involves only the positions of the particles rather than their orientations or types. In fact, positional order has been established for some models without internal degrees of freedom-in one dimension, ${ }^{(14)}$ in the Kac-van der Waals limit, ${ }^{(15,17)}$ and recently for certain long-range interactions by perturbation about this limit. ${ }^{(16)}$

In this paper we ask whether a direct interplay of positional and orientational order can be observed in specific situations. ${ }^{3}$ A physical picture illustrating an interplay between positions and orientations is the following. Consider a ferrofluid with a ferromagnetic spin interaction which decreases with the distance of the particles. A ferromagnetic ordering then induces an effective increase of the indirect attractive forces between the particles. This effect should increase the particle density. By the monotonicity of the spin coupling, the resulting lowering of the average particle distance implies an increase of the effective spin couplings, and thereby a strengthening of the ferromagnetic order. This in turn increases the particle density again, and so on. In thermodynamic terms, this means that certain values of the particle density and of the magnetization are impossible, so that these quantities must exhibit a jump. In other words, one expects that a direct feedback between the positional and the orientational structure of a system can change the nature of a phase transition from second order to first order.

${ }^{3}$ This question, of course, does no refer to the trivial fact that positional order in one system can be the consequence of orientational order in another system. This is well-known to be the case for the single-type Widom-Rowlinson model, which is the one-type marginal of the two-types Widom-Rowlinson model. ${ }^{(29)}$ 
It is the aim of the present paper to justify the above heuristics in a specific model. Since more realistic systems seem to be out of reach presently, we consider a toy model of mean-field type. Namely, we consider a system of classical particles with Ising or Heisenberg spins which are coupled by a ferromagnetic Curie-Weiss interaction. The point is that the exchange rate is inversely proportional to the volume rather than the particle number (with factor $J>0$ ), so that the effective field acting on each spin is proportional to the magnetization per volume rather than per particle. This allows for a feedback between the ferromagnetic and positional features of the model. The spin-independent interaction will be modelled by a suitable "phenomenological" function $g$ of the particle density. The shape of $g$ and its relation to $J$ determine the interplay of molecular and ferromagnetic forces. In addition to these constituents of the model we have, of course, the standard parameters $\beta>0$, the inverse temperature, and $z>0$, the activity or "a priori particle density."

We will show that, for suitable choices of these quantities, the model exhibits a first-order phase transition with simultaneous jumps of particle density and magnetization. This holds even if the direct particle interaction $g$ alone does not induce a phase transition. On the other hand, a densityindependent spin-coupling would only lead to a second-order transition. It is thus clear that the first-order phase transition is indeed a consequence of some feedback mechanism. If the molecular interaction $g$ already gives rise to a liquid-vapor transition, the interplay of positional and orientational order will create an even richer phase diagram containing a tricritical point.

We will now describe our results in some more detail. For simplicity we assume here that the particles have Ising spins with values \pm 1 . The ferromagnetic coupling constant $J>0$ is kept fixed, and we look for the phase diagram in the $(z, \beta)$-quadrant. The first basic fact is the existence of a continuous curve $z=z_{m}(\beta)$ which separates the nonmagnetic and the ferromagnetic parameter regions: The limiting phases of our system are nonmagnetic when $z<z_{m}(\beta)$ and ferromagnetic when $z>z_{m}(\beta)$. Whether or not the phase transition at $z_{m}(\beta)$ is of first order (with jumps of both density and magnetization) depends on the specific features of $g$. We therefore sketch three scenarios which exemplify the variety of possibilities. (For stability reasons we always assume that $g$ grows faster than quadratically.)

Scenario A. While the ferromagnetic phase transition at $z=z_{m}(\beta)$ is only of second order for small $\beta$, it is of first order when $\beta$ is sufficiently large.

This scenario occurs if $g^{\prime \prime}$ is increasing with $0 \leqslant 2 g^{\prime \prime}(0)<J$; cf. Proposition 3.2 and Theorem 3.3 below. The simplest example is $g(\rho)=c \rho^{3}$ with $c>0$; see Fig. 1 . 


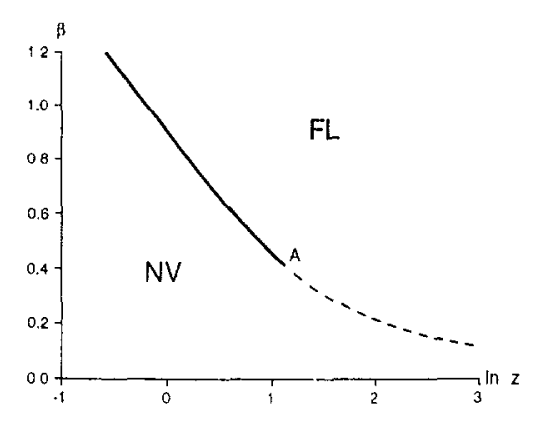

(a)

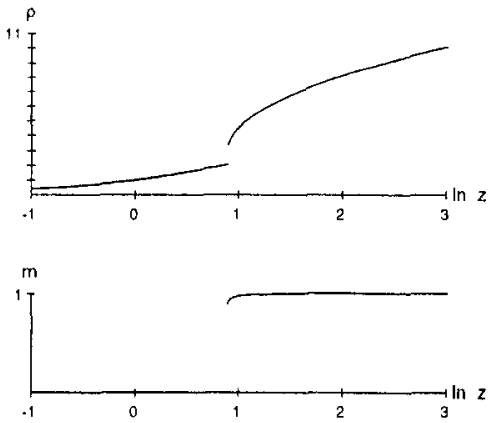

(b)

Fig. 1. The case $J=1, g(p)=\rho^{3} / 30$. (a) Phase diagram. The ferromagnetic transition curve $z=z_{m}(\beta)$ splits into two parts: a bold part indicating a first-order phase transition between nonmagnetic vapor (NV) and ferromagnetic liquid (FL), and a broken part corresponding to a second-order transition at high temperatures. The point $\mathrm{A}$ is the liquid-vapor critical point; the associated inverse temperature $\beta_{A}$ is determined by the equation $2 g^{\prime \prime}\left(1 / \beta_{A} J\right)=J$. (b) Density $(\rho)$ and magnetization per particle $(\mathrm{m})$ for $\beta=0.5$.

Scenario B. The ferromagnetic phase transition at $z=z_{m}(\beta)$ is still of second order for small $\beta$ and of first order for intermediate values of $\beta$. For large $\beta$, however, the phase transition at the magnetization threshold $z_{m}(\beta)$ is only of second order, while a first-order transition with simultaneous jumps of density and magnetization occurs at some $z>z_{m}(\beta)$, i.e., in the interior of the ferromagnetic parameter region.

This holds, for example, if $g^{\prime \prime}$ is convex with minimum 0 at some $\rho_{\text {min }}>0$, and $2 g^{\prime \prime}(0)>J$; see Corollary 3.6 and Theorem $3.4(\mathrm{c})$. A typical example is $g(\rho)=c(\rho-1)^{4}$ with $c>J / 24$; cf. Fig. 2 .

Scenario C. For some critical inverse temperature $\beta_{c}$ and $z_{c}=$ $z_{m}\left(\beta_{c}\right)$, there exist phases with three different densities $\rho_{-}<\rho_{\sharp}<\rho_{+}$. The phases with density $\rho_{+}$are ferromagnetic (with positive or negative orientation). The triple point $\left(z_{c}, \beta_{c}\right)$ is the endpoint of a first-order (liquidvapor) transition line in the (nonmagnetic) region $\left\{z<z_{m}(\beta), \beta<\beta_{c}\right\}$; see Fig. 3. Following this line towards $\left(z_{c}, \beta_{c}\right)$ one obtains the limiting densities $\rho_{-}$and $\rho_{\sharp}$. On the other hand, in a neighborhood of the triple point the ferromagnetic transition at the line $z=z_{m}(\beta)$ is also of first order. Approaching $\left(z_{c}, \beta_{c}\right)$ along this line from below $\left(\beta<\beta_{c}\right)$ one arrives at the limiting densities $\rho_{\#}$ and $\rho_{+}$, and coming from above $\left(\beta>\beta_{c}\right)$ one ends up with $p_{-}$and $\rho_{+}$. In other words, for $\beta \lesssim \beta_{c}$ there are two density jumps at 


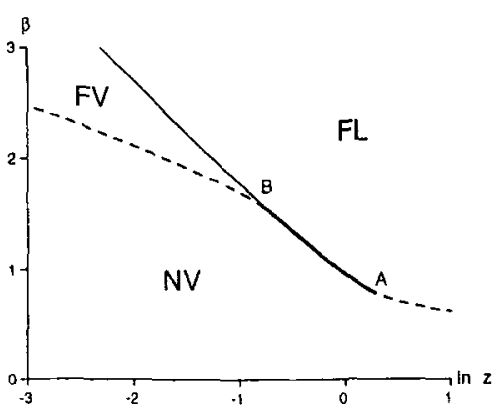

(a)
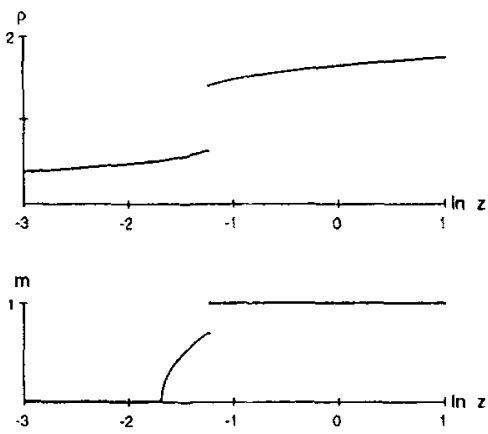

(b)

Fig. 2. The case $J=1, g(\rho)=(\rho-1)^{4}$. (a) Phase diagram. The bold and broken curves and the critical point $A$ have the same meaning as in Fig. 1. In addition to the two phases NV and $\mathrm{FL}$, there is an intermediate phase FV (ferromagnetic vapor); the solid line separating FV from FL indicates a first-order phase transition. Since the transition from NV to FV is second order, the critical point $B$ is not a triple point, $(b)$ Density and magnetization for $\beta=2$.

different activities, one due to the molecular forces and one at the incipience of ferromagnetic order. These jumps add up at $\beta=\beta_{c}$, and an enhanced jump persists for $\beta \gtrsim \beta_{\text {c. }}$.

This scenario occurs, for instance, if $g^{\prime \prime}$ is convex with $\min g^{\prime \prime}<0$, and $J$ is suitably chosen; see Theorem 3.8 and the example in Fig. 3.

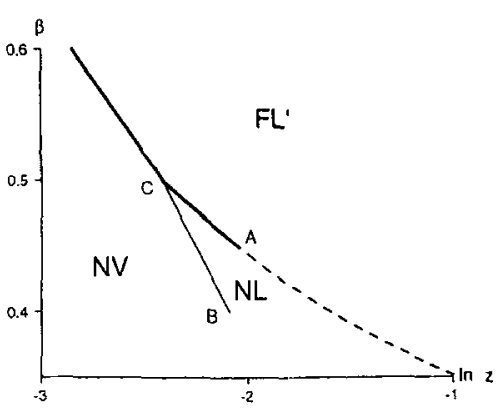

(a)

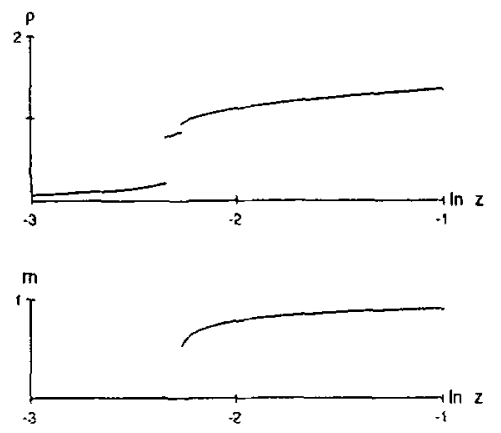

(b)

Fig. 3. The case $J=2.5, g(\rho)=\rho^{4}-4 \rho^{2}$. (a) Phase diagram. Bold and broken lines are as in Fig. 1. The solid line indicates a first-order vapor-liquid transition within the nonmagnetic region. The point $C$ is a triple point of coexistence of three phases: nonmagnetic vapor (NV), nonmagnetic liquid ( $N L$ ), and a ferromagnetic high-density phase which should probably also be interpreted as a liquid ( $\left.F L^{\prime}\right)$. $B$ is the V-L critical point, and A the L-L' critical point. (b) Density and magnetization for $\beta=0.48$. 
Although in the pictures above it is evident that there exist critical temperatures separating the different regimes of phase transition, it seems to be difficult to find satisfactory general criteria for this to be the case. We therefore concentrate on qualitative statements for large or small $\beta$.

The paper is organized as follows. In Section 2 we will introduce the model. Our results are stated in Section 3. We will use a general result from large deviation theory for marked point processes which allows us to express the infinite-volume behavior of our model in terms off the minimizers of a free energy functional. ${ }^{(10)}$ The necessary details will be provided in Section 4. Section 5 contains a general analysis of these minimizers. The proofs of our main results then follow in Section 6. In the final Section 7 we comment on the physical significance of our results.

\section{THE MODEL}

We consider a system of particles in Euclidean space ${ }^{4} \mathbf{R}^{d}, d \geqslant 1$. Each particle is equipped with a spin taking values in the unit sphere $E=$ $\left\{s \in \mathbf{R}^{D}:|s|=1\right\}$ of $\mathbf{R}^{D}, D \geqslant 1$. A configuration of such particles (without multiple occupancies) can be described by a pair $\omega=\left(X,\left(\sigma_{x}\right)_{x \in X}\right)$, where $X \subset \mathbf{R}^{d}$, the set of occupied positions, is locally finite, in that its intersection with any bounded set is finite, and $\sigma_{x} \in E$ is the spin of the particle at position $x$. Equivalently, one can think of $\omega$ as a locally finite subset of $\mathbf{R}^{d} \times E$ which has at most one point in each section $\{x\} \times E, x \in \mathbf{R}^{d}$. We write $\Omega$ for the set of all such configurations. $\Omega$ will be endowed with the smallest $\sigma$-algebra for which the counting variable $N(B): \omega \rightarrow \sum_{x \in X} 1_{\left\{\left(x, \sigma_{x}\right) \in B\right\}}$ is measurable for any Borel subset $B$ of $\mathbf{R}^{d} \times E$.

The a priori probability measure on $\Omega$ is the Poisson point random field describing an ideal gas of particles at random positions with random spins in $E$. Specifically, let $z>0$ be an activity parameter and $\mu$ an arbitrary Borel probability measure on $E$. The Poisson point random field $Q^{z \mu}$ with intensity $z$ and spin distribution $\mu$ is then defined as the unique probability measure on $\Omega$ such that, for any measurable function $f: \Omega \rightarrow[0, \infty$ [ which depends only on the configuration of the particles in a box $A \subset \mathbf{R}^{d}$ of finite volume $|\Lambda|$,

$$
\begin{aligned}
\int f d Q^{z \mu}= & e^{-z|A|} \sum_{k=0}^{\infty} \frac{z^{k}}{k !} \int_{\Lambda^{k}} d x_{1} \cdots d x_{k} \\
& \times \int_{E^{k}} \mu\left(d \sigma_{1}\right) \cdots \mu\left(d \sigma_{k}\right) f\left(\left\{\left(x_{1}, \sigma_{1}\right), \ldots,\left(x_{k}, \sigma_{k}\right)\right\}\right)
\end{aligned}
$$

${ }^{4}$ This choice is merely for simplicity. As we are going to consider a mean field model, the dimension $d$ and the Euclidean structure of $\mathbf{R}^{d}$ will not play any role. 
In particular, $z$ is the expected particle density of $Q^{z \mu}$, and conditionally on the set of occupied positions the spins are i.i.d. with distribution $\mu$. (Note that $Q^{z \mu}$ depends on $z$ and $\mu$ only through the finite measure $z \mu$, the spin intensity measure. So, in fact we have defined $Q^{v}$ for any finite measure $v$ on $E$.)

We will consider the following choices of $\mu$. A priori, we will set $\mu=\lambda$, the normalized surface measure on $E$. A posteriori, it will become necessary to consider, for each external field $\mathbf{h} \in \mathbf{R}^{D}$, the tilted probability measure

$$
\lambda_{\mathbf{h}}(d \sigma)=e^{\mathbf{h} \cdot \sigma} \lambda(d \sigma) / \int e^{\mathbf{h} \cdot \sigma} \lambda(d \sigma)
$$

on $E$. We write $Q^{z, \mathbf{h}}=Q^{z \lambda_{\mathbf{h}}}$. In particular, $Q^{z, 0}=Q^{z \lambda}$. Finally, we introduce the finite boxes $A_{n}=[-n, n]^{d}$ of volume $V_{n}=\left|A_{n}\right|, n \geqslant 1$, and we write $Q_{n}^{z, \mathbf{h}}$ for $Q^{z, \mathbf{h}}$ restricted to $A_{n}$, i.e., $Q_{n}^{z, \mathbf{h}}$ is the image of $Q^{z, \mathbf{h}}$ under the restriction mapping $\omega=\left(X,\left(\sigma_{x}\right)_{x \in X}\right) \rightarrow \omega_{n}=\left(X \cap A_{n},\left(\sigma_{x}\right)_{X \cap A_{n}}\right)$. Hence, $Q_{n}^{z, \mathbf{h}}$ is a probability measure on $\Omega_{n}=\left\{\omega=\left(X,\left(\sigma_{x}\right)_{x \in X}\right) \in \Omega: X^{n} \subset A_{n}\right\}$.

We consider a particle system in $A_{n}$ with an interaction of Curie-Weiss type. More precisely, for $\omega=\left(X,\left(\sigma_{x}\right)_{x \in X}\right) \in \Omega_{n}$ we consider the Hamiltonian

$$
H_{n}(\omega)=-\frac{J}{2} \sum_{x, y \in X} \frac{\sigma_{x} \cdot \sigma_{y}}{V_{n}}+V_{n} g\left(\frac{\# X}{V_{n}}\right)
$$

where $J>0$ is a ferromagnetic coupling constant, $g:[0, \infty[\rightarrow \mathbf{R}$ is a suitable function describing the molecular mean-field interaction, and $\# X$ is the cardinality of $X$. The essential features of this Hamiltonian are the following.

The spin coupling constant is proportional to $1 / V_{n}$ rather than $1 / \# X$. Hence, the mean field acting on a single spin is proportional to the particle density $\# X / V_{n}$ and the magnetization per particle. This favors high particle densities, and the system would become unstable without an additional term in the Hamiltonian. This is a formal reason for introducing the term with $g$. In addition, we want to ensure that $g$ gives rise to a positive feedback of the magnetization to the particle density. It is our objective to find the relevant features of $g$ for this to occur.

Our general assumptions on $g$ are the following. exists.

(A1) Smoothness. $g$ is analytic on $] 0, \infty\left[\right.$, and $g^{\prime \prime}(0)=\lim _{\rho \downarrow 0} g^{\prime \prime}(\rho)$

(A2) Strong stability. $g^{\prime \prime}(\rho) \rightarrow \infty$ as $\rho \rightarrow \infty$, and thus $g(\rho) / \rho^{2} \rightarrow \infty$ as $\rho \rightarrow \infty$. 
For example, $g$ can be any polynomial of degree at least three with positive leading coefficient. By the way, for a polynomial $g(\rho)=\sum_{k=1}^{K} a_{k} \rho^{k}$ we can write

$$
V_{n} g\left(\frac{\# X}{V_{n}}\right)=\sum_{k=1}^{K} \sum_{x_{1}, \ldots, x_{k} \in X} \frac{a_{k}}{V_{n}^{k-1}}
$$

which means that also the molecular part of $H_{n}$ is given by a many-body interaction of Curie-Weiss type. We might also require that the $x_{1}, \ldots, x_{k}$ in the sum above are pairwise distinct because this condition becomes irrelevant in the thermodynamic limit.

Let

$$
P_{n, z, \beta, J}(d \omega)=Z_{n, z, \beta, J}^{-1} e^{-\beta H_{n}(\omega)} Q_{n}^{z, 0}(d \omega)
$$

be the Gibbs distribution in $A_{n}$ with Hamiltonian $H_{n}$, activity $z>0$ and inverse temperature $\beta>0$. As usually,

$$
Z_{n, z, \beta, J}=\int e^{-\beta H_{n}(\omega)} Q_{n}^{z, 0}(d \omega)
$$

is the associated partition function. (Here and below we consider $g$ and $D$ as fixed, but the other parameters may vary and are thus included into our notation.)

We are interested in the set $\mathscr{G}(z, \beta, J)$ of all limiting Gibbs states, i.e., of all accumulation points of the sequence $\left(P_{n, z, \beta, J}\right)_{n \geqslant 1}$ in the infinitevolume limit $n \rightarrow \infty$. To make this definition precise we need to specify a topology for probability measures on $\Omega$. Rather than the usual weak topology, we can use here a finer topology $\tau_{\mathscr{L}}$ which is defined as follows. Let $\mathscr{L}$ be the class of all measurable functions $f: \Omega \rightarrow \mathbf{R}$ which are local, in that $f(\omega)$ only depends on the restriction of $\omega$ to a bounded box $A \subset \mathbf{R}^{d}$, and such that, for some $c<\infty,|f(\omega)| \leqslant c(1+\#(X \cap A))$ for all $\omega=\left(X,\left(\sigma_{x}\right)_{x \in X}\right) \in \Omega$. The topology $\tau_{\mathscr{L}}$ is then defined as the smallest topology relative to which all integral mappings $P \rightarrow \int f d P$ with $f \in \mathscr{L}$ are continuous. Note that the mean particle number in any region is $\tau_{\mathscr{L}}$-continuous as a function of the measure.

Theorem 4.1 below will imply that the sequence $\left(P_{n, z, \beta, J}\right)_{n \geqslant 1}$ is relatively sequentially compact in $\tau_{\mathscr{L}}$. Hence $\mathscr{G}(z, \beta, J)$ is always nonempty, and stating that $\mathscr{G}(z, \beta, J)$ is a singleton is equivalent to saying that $P_{n, z, \beta, J}$ converges, even in $\tau_{\mathscr{L}}$, towards the unique element of $\mathscr{G}(z, \beta, J)$. 


\section{RESULTS}

Our first theorem describes the general features of the set of all limiting Gibbs states.

Theorem 3.1. For any $z, \beta, J>0$, there is a finite set $\mathscr{M}(z, \beta, J) \subset$ ]0, $\infty$ [ such that $\mathscr{G}(z, \beta, J)$ consists of mixtures of the measures $P_{\rho, \beta J}$ with $\rho \in \mathscr{M}(z, \beta, J)$, where

$$
P_{\rho, \beta J}= \begin{cases}Q^{\rho, 0} & \text { if } \rho \leqslant D / \beta J \\ \int_{E} Q^{\rho, h_{*}(\beta J \rho) u} \lambda(d u) & \text { otherwise }\end{cases}
$$

The function $h_{*}(\beta J \rho)$ above (which depends on $D$ only and is defined implicitly as the largest solution of the mean field equation (14)) is positive and strictly increasing for $\beta J \rho>D$. The set $\mathscr{M}(z, \beta, J)$ can be identified as the set of all minimizers of a suitable variational functional and exhibits, in particular, the following properties.

(i) For fixed $\beta, J$ there are at most countably many values of $z$ for which $\mathscr{M}(z, \beta, J)$, and thus $\mathscr{G}(z, \beta, J)$, is not a singleton.

(ii) $\mathscr{A}(z, \beta, J)$ depends monotonically on $z$, in that $\rho<\rho^{\prime}$ whenever $z<z^{\prime}$ and $\rho \in \mathscr{M}(z, \beta, J), \rho^{\prime} \in \mathscr{M}\left(z^{\prime}, \beta, J\right)$. Also, $\min \mathscr{M}(z, \beta, J) \rightarrow \infty$ as $z \rightarrow \infty$ and $\max \mathscr{M}(z, \beta, J) \rightarrow 0$ as $z \rightarrow 0$.

(iii) The graph of the correspondence $(z, \beta, J) \rightarrow \mathscr{M}(z, \beta, J)$ is closed.

Several remarks are in order.

Remarks. (a) The limiting Gibbs state $P_{\rho, \beta J}$ describes a Poissonian nonmagnetic phase when $\rho \leqslant D / \beta J$, and a uniform mixture of Poissonian magnetic phases when $\rho>D / \beta J$. Moreover, it follows from property (ii) above that for any $\beta, J>0$ there is a unique $\left.z_{m}=z_{m}(\beta, J) \in\right] 0, \infty[$ such that the limiting Gibbs states in $\mathscr{G}(z, \beta, J)$ are nonmagnetic (with density $\rho<D / \beta J$ ) when $z<z_{m}$ and ferromagnetic (with density $\rho>D / \beta J$ ) when $z>z_{m} . z_{m}(\beta, J)$ is therefore the critical activity for magnetization. We will see later that $z_{m}$ depends continuously on $\beta$ and $J$ and is strictly decreasing in $J$.

(b) For any parameter triple $(z, \beta, J)$, a first-order phase transition occurs if and only if $\mathscr{M}(z, \beta, J)$ is not a singleton. We then have a jump of the particle density and of the magnetization per particle. An interesting particular case occurs when $\mathscr{M}(z, \beta, J)$ contains two elements $\rho_{-}, \rho_{+}$with $\rho_{-}<D / \beta J<\rho_{+}$, which is only possible for $z=z_{m}(\beta, J)$. Then there are a 
nonmagnetic phase of density $\rho_{-}$and magnetic phases of strictly larger density $\rho_{+}$with arbitrary orientations. This corresponds to a discontinuous appearance of magnetization which is coupled to a vapor-liquid transition.

(c) To keep things as simple as possible we did not introduce an external field into our Hamiltonian (2). This is the obvious reason why the limiting Gibbs measures $P_{\beta, \beta J}$ above are uniform mixtures of magnetic phases when $\beta J \rho>D$. Introducing external fields which tend to 0 sufficiently slowly as $n \rightarrow \infty$ and letting also $z$ vary with $n$ we could obtain a particular magnetic phase, or a particular mixture, as limiting Gibbs state, cf. ref. 1 . We leave this to the interested reader.

We now turn to a description of various scenarios for a first-order transition. To begin, we note that such a transition neither occurs at high temperatures nor for weak ferromagnetic coupling, provided such a transition is not already induced by the molecular interaction $g$ alone-which can be excluded by a convexity assumption on $g$.

Proposition 3.2 (Absence of density jumps). Suppose that either

(i) $g$ and $J$ are arbitrarily given and $\beta>0$ is sufficiently small; or

(ii) $g$ is convex, $\beta$ is fixed, and $J \geqslant 0$ is sufficiently small.

Then $\mathscr{M}(z, \beta, J)$ is a singleton for all $z>0$.

Next we state a simple sufficient condition for a first-order transition from a nonmagnetic vapor to a magnetic liquid at $z=z_{m}(\beta, J)$ according to Scenario A of the introduction. This condition holds in particular when $g$ is convex, i.e., if (by Proposition 3.2) there is no first-order phase transition for vanishing magnetic coupling $J=0$. In this case, the jump of density and magnetization is not induced by the molecular forces alone, but comes from their interplay with the ferromagnetic forces.

Theorem 3.3 (First-order transition from nonmagnetic vapor to ferromagnetic liquid). Suppose $\beta, J>0$ are such that $J>2 g^{\prime \prime}(D / \beta J)$. (Evidently, this holds when either $\beta>0$ is arbitrarily fixed and $J$ is sufficiently large, or $J>2 g^{\prime \prime}(0)$ is given and $\beta$ is sufficiently large.) Then, for $z=z_{m}(\beta, J), \mathscr{M}(z, \beta, J)$ contains two densities $\rho_{-}, \rho_{+}$with $\rho_{-}<D / \beta J<\rho_{+}$.

A fairly complete picture can be obtained in the low-temperature and strong-coupling limits. The low-temperature limit depends on pieces of non-convexity of the function $g(\rho)-J \rho^{2} / 2$, as is specified in the next definition. 
Definition. Let $f:[0, \infty[\rightarrow \mathbf{R}$ be continuous and bounded from below, and let $f^{* *}$ denote its convex envelope. We will say a nondegenerate interval $\left[r_{-}, r_{+}\right]$is critical for $f$ with slope $\gamma$ if

(i) $f^{* *}<f$ on $] r_{-}, r_{+}[$

(ii) for some $\varepsilon>0, f^{* *}$ and $f$ coincide and are strictly convex on $\left[r_{+}, r_{+}+\varepsilon\right]$ and, if $r_{-}>0$, also on $\left[r_{-}-\varepsilon, r_{-}\right]$; and

(iii) $\gamma$ is the slope of $f^{* *}$ on $\left[r_{-}, r_{+}\right]$.

Theorem 3.4 (Low temperature behavior). Let $J \geqslant 0$ be fixed. Suppose $\left[r_{-}, r_{+}\right]$is any critical interval of the function $g_{J}(\rho)=g(\rho)-J \rho^{2} / 2$, and $\gamma$ is the associated slope. Then the following is true.

(a) For any $\varepsilon>0$ and sufficiently large $\beta$, there exists a unique activity $z$ with $\left|\beta^{-1} \ln z-\gamma\right|<\varepsilon$ such that $\mathscr{M}(z, \beta, J)$ contains two distinct elements $\rho_{-}, \rho_{+}$satisfying $\left|\rho_{-}-r_{-}\right|<\varepsilon$ and $\left|\rho_{+}-r_{+}\right|<\varepsilon .\left(z, \rho_{-}\right.$and $\rho_{+}$ depend on $\beta, \varepsilon$, the critical interval $\left[r_{-}, r_{+}\right]$and, of course, $J, g$, and $D$.)

(b) Suppose further that $g_{J}^{\prime \prime}\left(r_{+}\right)>0$ and either, if $r_{-}>0, g_{J}^{\prime \prime}\left(r_{-}\right)>0$ or, if $r_{-}=0, g_{J}^{\prime}(0)>\gamma$. If $\beta$ is large enough and $z$ is as in (a) then $\mathscr{M}(z, \beta, J)=\left\{\rho_{-}, p_{+}\right\}$.

(c) Suppose, in addition, that $g_{J}^{\prime \prime}>0$ on $\left[r_{+}, \infty\right.$ [ and, if $r_{-}>0$, on $\left[0, r_{-}\right]$, so that $\left[r_{-}, r_{+}\right]$is the only critical interval of $g_{J}$. In the case $r_{-}>0$, we need to assume further that $D=1$ or $g_{J}^{\prime \prime}(0)>J(b(D)-1)$ for the constant ${ }^{5} b(D)$ defined by (19). Then, for sufficiently large $\beta$, the activity $z$ in (a) is the unique value of $z$ for which $\mathscr{M}(z, \beta, J)$ is not a singleton.

The behavior for large magnetic coupling is simpler because it is independent of the shape of $g$.

Theorem 3.5 (Strong ferromagnetic coupling). Let $\beta>0$ be given and $\varepsilon>0$. If $J$ is sufficiently large, there exists some $z>0$ with $J^{-1} \ln z<$ $-\varepsilon^{-1}$ such that $\mathscr{M}(z, \beta, J)$ contains two densities $\rho_{-}$and $\rho_{+}$satisfying $\rho_{-}<\varepsilon$ and $\rho_{+}>\varepsilon^{-1}$. For $D=1$ and any other $D$ for which Lemma $5.2(\mathrm{~g})$ holds we have $\mathscr{M}(z, \beta, J)=\left\{\rho_{-}, \rho_{+}\right\}$, and $\# \mathscr{M}\left(z^{\prime}, \beta, J\right)=1$ for all $z^{\prime} \neq z$.

As we have claimed in Scenario B of the Introduction it can happen that, for large $\beta$, the ferromagnetic transition at $z_{m}$ is of second order but, at some $z>z_{m}$, a jump of both density and magnetization occurs. This is the subject of the next corollary which applies to convex functions like $g(\rho)=(\rho-1)^{4}$.

\footnotetext{
${ }^{5}$ Numerical calculations suggest that $b(D)=1$ for $D \geqslant 2$, so that the additional assumption is redundant. But we could not prove this.
} 
Corollary 3.6 (First-order transition between ferromagnetic phases). Suppose $g^{\prime \prime}$ attains its minimum 0 at a single point $\rho_{\min }>0$. If $J>0$ is sufficiently small and $\beta$ is sufficiently large, there is then a unique $z>z_{m}(\beta, J)$ such that $\mathscr{M}(z, \beta, J)=\left\{\rho_{-}, \rho_{+}\right\}$with $D / \beta J<\rho_{-}<\rho_{\min }<\rho_{+}$.

Next we consider the case when $g$ is not convex, which means that the molecular forces alone already imply a first-order phase transition when $\beta$ is large enough. For large $J$, Theorem 3.3 asserts that there is a discontinuous transition to ferromagnetic order at $z=z_{m}(\beta, J)$. The next proposition shows that, for small $J$, the density jumps induced by $g$ do not imply a ferromagnetic ordering, so that the magnetization depends continuously on $z$. (This statement may be viewed as a counterpart to Proposition 3.2(ii) for non-convex $g$.)

Proposition 3.7 (First-order transition between non-magnetic phases). Suppose that $\min _{\rho>0} \rho g^{\prime \prime}(\rho) \equiv-1 / \beta_{\min }<0, \beta>\beta_{\min }$, and $J$ is sufficiently small. Then there exists a unique $0<z<z_{m}(\beta, J)$ such that $\mathscr{M}(z, \beta, J)=$ $\left\{\rho_{-}, \rho_{+}\right\}$with $\rho_{-}<\rho_{+}<D / \beta J$, and $\# \mathscr{M}\left(z^{\prime}, \beta, J\right)=1$ whenever $z^{\prime}>z$. In particular, the ferromagnetic phase transition at $z_{m}(\beta, J)$ is of second order.

Our final result provides conditions for the existence of two first-order transition lines which join at a triple point with three different phases. The first of these transition lines comes frogs the molecular forces alone, whereas the second is again the result of a feedback between molecular and magnetic forces. After the joining at the triple point, one has the same picture as in Theorem 3.3-a discontinuous incipience of ferromagnetic order at $z=z_{m}(\beta, J)$ together with a jump of density which is partly due to the molecular forces but enhanced by the ferromagnetic spin-interaction.

Theorem 3.8 (Existence of a triple point with phases of three different densities). Suppose there exists some unique $\rho_{\text {min }}>0$ such that

$$
\rho_{\min } g^{\prime \prime}\left(\rho_{\min }\right)=\min _{\rho>0} \rho g^{\prime \prime}(\rho) \equiv-1 / \beta_{\min }<0
$$

If $\beta>\beta_{\min }$ is sufficiently close to $\beta_{\min }$, there exist unique numbers $z_{c}=z_{c}(\beta)>0$ and $J_{c}=J_{c}(\beta)>0$ such that $\mathscr{M}\left(z_{c}, \beta, J_{c}\right) \supset\left\{\rho_{-}, \rho_{\sharp}, \rho_{+}\right\}$, where $0<\rho_{-}<\rho_{\#}<D / \beta J_{c}<\rho_{+}$. At least if $D=1$ and $g^{\prime \prime}$ is convex, we have $\mathscr{M}\left(z_{c}, \beta, J_{c}\right)=\left\{\rho_{-}, \rho_{\#}, \rho_{+}\right\}$. In this case we can also state, writing $z_{J}=z_{m}(\beta, J)$ :

(a) If $J>J_{c}$ and $J$ is sufficiently close to $J_{c}$ then $z_{J}<z_{c}, z_{J}$ is close to $z_{c}$, and $\mathscr{M}\left(z_{J}, \beta, J\right)$ contains two distinct elements arbitrarily close to $\rho_{-}$resp. $\rho_{+}$. 
(b) If $J<J_{c}$ and $J$ is sufficiently close to $J_{c}$ then $\rho_{-}, \rho_{\sharp} \in$ $\mathscr{M}\left(z_{c}, \beta, J\right), z_{J}>z_{c}, z_{J}$ is close to $z_{c}$, and $\mathscr{M}\left(z_{J}, \beta, J\right)$ contains two distinct elements arbitrarily close to $\rho_{\#}$ resp. $\rho_{+}$.

It will be evident from the proof that if $g$ alone already admits three or more nonmagnetic phases at some $\beta$ then, for suitable $J=J_{c}(\beta)$, the spin-interaction leads to the existence of additional phases of higher density. This implies the existence of quadruple points, and so on.

The proof will also show that $J_{c}(\beta)$ is continuous and strictly monotone whenever the critical activity for the density jump induced by $g$ alone is a strictly monotone function of $\beta$--which can be checked in special cases. Then it is possible to replace the independent parameter $J$ in Theorem 3.8 by $\beta$, and we obtain a statement as in Scenario $C$ of the Introduction.

Finally we note that the first-order transition line described in statement (a) may split at some critical point into two branches corresponding to a second-order transition to ferromagnetic order at $z=z_{m}$ and, on the other hand, to jumps of density and magnetization in the regime of positive magnetization. This does in fact occur in situations similar to those described in Corollary 3.6.

\section{THE MAXIMUM ENTROPY PRINCIPLE}

Theorem 3.1 will be derived from a more general conditional limit theorem which was obtained in ref. 10. In this section we will provide the necessary details. (We might replace $E$ by an arbitrary Polish space in the following, but for simplicity we stick to the setting of the previous sections.)

Let $\mathscr{M}_{E}$ denote the space of all finite Borel measures on $E$. $\mathscr{M}_{E}$ will be equipped with the smallest topology which is such that, for any bounded measurable function $f$ on $E$, the evaluation map $v \rightarrow \int f d v$ is continuous. The relative entropy of two measures $\mu, v \in \mathscr{M}_{E}$ is defined by

$$
I(v ; \mu)= \begin{cases}\int(1-f+f \ln f) d \mu & \text { if } v \ll \mu \text { with density } f \\ \infty & \text { otherwise }\end{cases}
$$

It is well-known and easy to see that $I(v ; \mu) \geqslant 0$ with equality if and only if $v=\mu$. Moreover, for any fixed $\mu$ and $c \geqslant 0$ the sublevel-set $\left\{v \in \mathscr{M}_{E}\right.$ : $I(v ; \mu) \leqslant c\}$ is compact, cf. ref. 10 . We will need the relative entropy in the special case when $\mu$ is a multiple of our a priori measure $\lambda$, and then use the notation $I_{z}(v)=I(v ; z \lambda)$ for $z>0$. 
The main quantity of interest is the empirical spin intensity measure of a configuration $\omega \in \Omega_{n}$ which is defined by

$$
L_{n, \omega}=V_{n}^{-1} \sum_{x \in X} \delta_{\sigma_{x}} \in \mathscr{M}_{E}
$$

where $\delta_{a}$ stands for Dirac measure at $a$. Its total mass is nothing other than the particle density, $L_{n, \omega}(E)=\# X / V_{n}$, and the normalized integral

$$
\int_{E} \sigma L_{n, \omega}(d \sigma) / L_{n, \omega}(E)=\frac{1}{\# X} \sum_{x \in X} \sigma_{x}
$$

is the average magnetization per particle.

The following theorem is a special case of Corollary 3.6 of ref. 10 . It is a point process counterpart of Sanov's large deviation principle and Csiszar's associated conditional limit theorem, cf. ref. 5.

Let $H: \mathscr{M}_{E} \rightarrow \mathbf{R}$ be a continuous functional such that $H(v) \geqslant-b v(E)$ for all $v \in \mathscr{M}_{E}$ and some $b<\infty$. We consider the local Hamiltonians

$$
H_{n}(\omega)=V_{n} H\left(L_{n, \omega}\right), \quad \omega \in \Omega_{n}
$$

and the associated partition functions $Z_{n, z, \beta, H}$ and Gibbs distribution $P_{n, z, \beta, H}$ defined in analogy to (4) and (3).

Theorem 4.1. For all $z, \beta>0$, the pressure

$$
p(z, \beta, H)=\lim V_{n}^{-1} \ln Z_{n, z, \beta, H}=-\min _{v \in \mathscr{M}_{E}}\left[I_{z}(v)+\beta H(v)\right]
$$

exists. Moreover, the sequence $\left(P_{n, z, \beta, H}\right)_{n \geqslant 1}$ is relatively sequentially compact in $\tau_{\mathscr{L}}$, and every accumulation point has the form $\int Q^{v} w(d v)$ for some Borel probability measure on the non-empty compact set $\left\{v \in \mathscr{M}_{E}: I_{z}(v)+\right.$ $\beta H(v)=-p(z, \beta, H)\}$.

In Example 4.2 of ref. 10, this theorem was applied to the functional $H(v)=-\left|\int \sigma v(d \sigma)\right|^{2} / 2 v(E)$ for which the Hamiltonian (7) is the direct continuum analog of the Curie-Weiss lattice model. It was shown that in this case a second-order phase transition occurs.

The model considered in this paper corresponds to the choice

$$
H(v)=-\frac{J}{2}\left|\int \sigma v(d \sigma)\right|^{2}+g(v(E))
$$


$v \in \mathscr{M}_{E}$. Indeed, defining $H_{n}(\omega)$ by (7) in terms of this $H$ we arrive at (2). To apply Theorem 4.1 we thus need to investigate the free energy functional $I_{z}+\beta H$. We note first that $H$ is obviously continuous. Also, $I_{z}+\beta H$ attains its minimum because $I_{z}$ has compact sublevel-sets. We will show that the minimizers are of the form $\rho \lambda_{\mathbf{h}}$ with suitable $\rho \geqslant 0, \mathbf{h} \in \mathbf{R}^{D}$.

To this end we first introduce the logarithmic moment generating function

$$
\varphi(h)=\ln \int e^{h \sigma_{1}} \lambda(d \sigma), \quad h \in \mathbf{R}
$$

of the first coordinate $\sigma_{1}$ of $\sigma$. Obviously, $\varphi$ is analytic, and the symmetry of $\lambda$ implies that, for all $\mathbf{h} \in \mathbf{R}^{D}$ and $h=|\mathbf{h}|$,

$$
\varphi(h)=\ln \int e^{\mathbf{h} \cdot \sigma} \lambda(d \sigma)
$$

and

$$
\varphi^{\prime}(h)=\left|\int \sigma \lambda_{\mathbf{h}}(d \sigma)\right|
$$

For $D=1, \varphi(h)=\ln \cosh h$ and $\varphi^{\prime}(h)=\tanh h$, whereas for $D=3$ we have $\varphi(h)=\ln (\sinh h / h)$ and $\varphi^{\prime}(h)=\operatorname{coth} h-h^{-1}$.

Next, we take an arbitrary $v \in \mathscr{M}_{E}$ and set $\rho=v(E)$. If $I_{z}(v)<\infty, v$ is not supported on a single point. So we can find some $\mathbf{h} \in \mathbf{R}^{D}$ such that $\int \sigma v(d \sigma)=\rho \int \sigma \lambda_{\mathbf{h}}(d \sigma)$. Equation (10) then shows that $H(v)=g(\rho)-$ $(J / 2) \rho^{2} \varphi^{\prime}(h)^{2}$, where again $h=|\mathbf{h}|$. In view of $(1), \rho \lambda_{\mathbf{h}}$ has density $u=(\rho / z) \exp [\mathbf{h} \cdot \sigma-\varphi(h)]$ with respect to $z \lambda$. Since $\int \ln u d v=\int \ln u d\left(\rho \lambda_{\mathbf{h}}\right)$, we conclude from (5) that $I_{z}(v)=I\left(v ; \rho \lambda_{\mathbf{h}}\right)+I_{z}\left(\rho \lambda_{\mathrm{h}}\right)$. On the other hand, using $(10)$ we also see that

$$
I_{z}\left(\rho \lambda_{\mathbf{h}}\right)=z\left[1-\frac{\rho}{z}+\frac{\rho}{z} \ln \frac{\rho}{z}\right]+\rho\left[h \varphi^{\prime}(h)-\varphi(h)\right]
$$

It is interesting to note that the first term on the right-hand side is simply the relative entropy of the Poisson distribution with parameter $\rho$ relative to the Poisson distribution with parameter $z$. The term in the second square bracket is equal to $\varphi^{*}(m)$, where $m=\varphi^{\prime}(h)$ is the magnetization corresponding to the external field $h$ and $\varphi^{*}(m)=\sup _{h \in \mathbf{R}}[h m-\varphi(h)]$, the Legendre-Fenchel transform of $\varphi$, is the so-called Cramér-transform of $\lambda$ which governs the large deviations of the particle spins. (From this it might 
seem natural to replace the parameter $h$ by $m$, but it turns out that $h$ is more convenient to work with.)

Combining the preceding calculations we find that

$$
I_{z}(v)+\beta H(v)=I\left(v ; \rho \lambda_{\mathbf{h}}\right)+F_{z, \beta, J}(\rho, h)
$$

where $h=|\mathbf{h}|$ and $F_{z, \beta, J}$ is given by

$$
\begin{aligned}
F_{z, \beta, J}(\rho, h) & =I_{z}\left(\rho \lambda_{\mathbf{h}}\right)+\beta H\left(\rho \lambda_{\mathbf{h}}\right) \\
& =z\left[1-\frac{\rho}{z}+\frac{\rho}{z} \ln \frac{\rho}{z}\right]+\rho\left[h \varphi^{\prime}(h)-\varphi(h)\right]+\beta\left[g(\rho)-\frac{J}{2} \rho^{2} \varphi^{\prime}(h)^{2}\right]
\end{aligned}
$$

By the properties of relative entropy, the right-hand side of (11) is minimal precisely for $v=\rho \lambda_{\mathbf{h}}$. We thus end up with the following conclusion.

Proposition 4.2. For the functional $H$ in (8) and any $z, \beta>0$, $I_{z}+\beta H$ attains its minimum on the set of all measures $\rho \lambda_{\mathbf{h}}$ for which $(\rho,|\mathbf{h}|)$ is a minimizer of $E_{z, \beta, J}$.

Since the measures $P_{n, z, \beta, J}$ in (3) are invariant under simultaneous rotations of all spins, the measure $w$ in Theorem 4.1 must also exhibit this rotational invariance. Hence, Theorem 3.1 will follow once we have identified the minimizers of the functional $F_{z, \beta, J}$. This is the subject of the next section.

\section{THE MINIMIZERS OF $\boldsymbol{F}_{z, \beta, J}$}

We start with some properties of the logarithmic moment generating function $\varphi$ in (9).

Lemma 5.1. (a) $\varphi$ is even, nonnegative and strictly convex and attains its minimum 0 at 0 .

(b) $\varphi^{\prime}$ is strictly concave on $\left[0, \infty\left[\right.\right.$. In particular, $\varphi^{\prime}(h) / h$ is strictly decreasing from $\varphi^{\prime \prime}(0)=1 / D$ to 0 as $h$ runs from 0 to $\infty$.

Proof. Since $\varphi^{\prime \prime}(h)$ is the variance of $\sigma_{1}$ under $\lambda_{\mathbf{l}}$ for $\mathbf{h}=(h, 0, \ldots, 0)$ and $\lambda_{\mathbf{h}}$ is nondegenerate, it is immediate that $\varphi$ is strictly convex. For $h=0$, the symmetry of $\lambda$ implies that $\varphi^{\prime \prime}(0)=\int \sigma_{i}^{2} \lambda(d \sigma)$ for each coordinate $i$. Averaging over $i$ we see that $\varphi^{\prime \prime}(0)=1 / D$. The strict concavity of $\varphi^{\prime}$ is proved in Theorem II.13.5 of ref. 23. 
In view of Lemma 5.1(b), the equation $h=a \varphi^{\prime}(h)$ with $a>0$ has a unique positive solution $h_{*}(a)>0$ if and only if $a>D$, whereas $h_{*}(a)=0$ is the only solution when $a \leqslant D$. The function $h_{*}(\cdot)$ has the following properties.

Lemma 5.2. (a) On $] D, \infty\left[, h_{*}(\cdot)\right.$ is strictly increasing and analytic.

(b) $h_{*}(a) \leqslant a$ for all $a>0$, and $h_{*}(a) / a \rightarrow 1$ as $a \rightarrow \infty$.

(c) $h_{*}(a) \sim \sqrt{D+2}(a-D)^{1 / 2}$ as $a \downarrow D$.

(d) $\varphi \circ h_{*}(a) \leqslant a$ for all $a>0$, and $\varphi \circ h_{*}(a) / a \rightarrow 1$ as $a \rightarrow \infty$.

(e) $\varphi \circ h_{*}(a) \sim[(D+2) / 2 D](a-D)$ as $a \downarrow D$.

(f) $h_{*}^{\prime}(a) \rightarrow 1$ and $\left(\varphi \circ h_{*}\right)^{\prime}(a) \rightarrow 1$ as $a \rightarrow \infty$.

(g) For $D=1,\left(\varphi \circ h_{*}\right)^{\prime}(a)-1<1 / a$ for all $a>1$.

(h) For $D=1,\left(\varphi \circ h_{*}\right)^{\prime}$ is decreasing on $] 1, \infty[$.

The proof will be postponed until the end of this section. Consider now the variational functional

$F_{z, \beta, J}(\rho, h)=z\left[1-\frac{\rho}{z}+\frac{\rho}{z} \ln \frac{\rho}{z}\right]+\rho\left[h \varphi^{\prime}(h)-\varphi(h)\right]+\beta\left[g(\rho)-\frac{J}{2} \rho^{2} \varphi^{\prime}(h)^{2}\right]$

introduced in (12). From Proposition 4.2 we know that $F_{z, \beta, J}$ attains its global minimum. We need to investigate the minimizers. In particular, we seek conditions under which the minimizer is not unique. To this end we first fix an arbitrary $\rho>0$ and minimize over $h$. By (12), we can assume that $h \geqslant 0$. Setting $\partial F_{z, \beta, J} / \partial h=0$ and recalling that $\varphi^{\prime \prime}(h)>0$ we arrive at the equation

$$
h=\beta J \rho \varphi^{\prime}(h)
$$

which is the usual mean field equation for the effective external field $h$ resp. the associated magnetization $\varphi^{\prime}(h)$. For $\beta J \rho \leqslant D$, this has the unique solution $h=0$. In the case $\beta J \rho>D, h=0$ is still a solution of $(13)$ but does not correspond to a minimum because then

$$
\left.\frac{\partial^{2}}{\partial h^{2}} F_{z, \beta, J}(\rho, h)\right|_{h=0}=\frac{\rho}{D}\left(1-\frac{\beta J \rho}{D}\right)<0
$$


We thus conclude that any global minimizer $(p, h)$ of $F_{z, \beta, J}$ satisfies the equation

$$
h=h_{*}(\beta J \rho)
$$

where (as above) $h_{*}(a)$ is the largest solution of the equation $h=a \varphi^{\prime}(h)$.

We are thus left with a minimization over the single parameter $\rho$, and we need to find the minimizers of the function $\rho \rightarrow F_{z, \beta, J}\left(\rho, h_{*}(\beta J \rho)\right)$. To this end we write

$$
F_{z, \beta, J}\left(\rho, h_{*}(\beta J \rho)\right)=z+G_{\beta, J}(\rho)-\rho \ln z
$$

where

$$
G_{\beta, J}(\rho)=\beta g(\rho)-\rho+\rho \ln \rho+\frac{\rho}{2} h_{*}(\beta J \rho) \varphi^{\prime} \circ h_{*}(\beta J \rho)-\rho \varphi \circ h_{*}(\beta J \rho)
$$

In view of Eq. (13) and its consequence for $h_{*}^{\prime}$, the derivative of $G_{\beta, J}$ exists and is given by

$$
G_{\beta, J}^{\prime}(\rho)=\ln \rho+\beta g^{\prime}(\rho)-\varphi \circ h_{*}(\beta J \rho)
$$

for all $\rho>0$ (including the singular value $\rho=D / \beta J$ ). By assumptions (A1) and (A2) and Lemma 5.2(d), we have $G_{\beta, J}^{\prime}(\rho) \rightarrow-\infty$ as $\rho \rightarrow 0$ and $G_{\beta, J}^{\prime}(\rho)$ $\rightarrow \infty$ as $\rho \rightarrow \infty$. Consequently, for each $c \in \mathbf{R}$ the function $c \rho-G_{\beta, J}(\rho)$ attains its maximum over $\rho$ on a compact subinterval of $] 0, \infty$, so that the Legendre-Fenchel transform

$$
G_{\beta, J}^{*}(c)=\sup _{\rho>0}\left[c \rho-G_{\beta, J}(\rho)\right]
$$

of $G_{\beta, J}$ is finite. $G_{\beta, J}^{*}$ is a convex, and thereby continuous, real function on $\mathbf{R}$. Geometrically, $G_{\beta, J}^{*}(c)$ is the smallest $a \in \mathbf{R}$ such that the straight line $\rho \rightarrow c \rho-a$ of slope $c$ does not exceed $G_{\beta, J}$. Consequently, $G_{\beta, J}^{*}$ coincides with the Legendre-Fenchel transform of the convex envelope $G_{\beta, J}^{* *}$ of $G_{\beta, J}$, which is defined as the largest convex function not exceeding $G_{\beta, J}$. Theorem 12.2 of ref. 21 therefore implies that the convex envelope of $G_{\beta, J}$ is indeed equal to the Legendre-Fenchel transform of $G_{\beta, J}^{*}$; this justifies our notation.

Let $\rho_{\beta, J,-}(z)$ and $\rho_{\beta, J,+}(z)$ be the left resp. right derivative of $G_{\beta, J}^{*}(c)$ at $c=\ln z$. By convexity, the set $D_{\beta, J}=\left\{z>0: \rho_{\beta, J,-}(z)<\rho_{\beta, J,+}(z)\right\}$ is at most countable. Now, it is well-known (Theorem 23.5 of ref. 21) and easy to see that, for each $z>0$, the function $G_{\beta, J}^{* *}(\rho)-\rho \ln z$ attains its global minimum precisely on the interval $\left[\rho_{\beta, J,-}(z), \rho_{\beta, J,+}(z)\right]$, and this means 
that $\rho_{\beta, J,-}(z)$ and $\rho_{\beta, J_{,}}(z)$ are the smallest resp. the largest global minimizer of $G_{\beta, J}(\rho)-\rho \ln z$. Vice versa, for any $\rho>0$ there is a unique $z$ such that $\rho \in\left[\rho_{\beta, J,-}(z), \rho_{\beta, J,+}(z)\right]$, namely $z=\exp \left(G_{\beta, J}^{* *}\right)^{\prime}(\rho)$. (Note that the last derivative exists because a kink in the graph of $G_{\beta, J}^{* *}$ would imply a kink in the graph of $G_{\beta, J}$, but this is impossible because $G_{\beta, J}$ is differentiable.) We thus end up with the following conclusion.

Proposition 5.3. For any $z, \beta, J>0$, the set of all global minimizers of $F_{z, \beta, J}$ is equal to $\left\{\left(\rho, h_{*}(\beta J \rho)\right): \rho \in \mathcal{M}(z, \beta, J)\right\}$, where

$$
\mathscr{M}(z, \beta, J)=\left\{\rho>0: G_{\beta, J}(\rho)=G_{\beta, J}^{* *}(\rho),\left(G_{\beta, J}^{* *}\right)^{\prime}(\rho)=\ln z\right\}
$$

is a finite set with convex hull $\left[\rho_{\beta, J,-}(z), \rho_{\beta, J,+}(z)\right]$. In particular, $\# \cdot \mathscr{H}(z, \beta, J)>1$ if and only if $G_{\beta, J}^{*}$ is not differentiable at $\ln z$.

Proof. It only remains to show that $\mathscr{M}(z, \beta, J)$ is finite. Since $\mathscr{H}(z, \beta, J)$ is contained in the set $\left\{\rho>0: G_{\beta, J}^{\prime}(\rho)=\ln z\right\}$, this follows from the analyticity assumption (A1) and Lemma 5.2(a).

It is evident that the sets $\mathscr{M}(z, \beta, J)$ exhibit the monotonicity property in z stated in Theorem 3.1(ii). The closed-graph property (iii) follows from the fact that $G_{\beta, J}, G_{\beta, J}^{* *}$ and, by convexity and differentiability, also $\left(G_{\beta, J}^{* *}\right)^{\prime}$ depend continuously on $(\beta, J)$. The proof of Theorem 3.1 is therefore complete. We also note that $z_{m}(\beta, J)$ is obviously equal to $\exp \left(G_{\beta, J}^{*}\right)^{\prime}(D / \beta J)$ and therefore continuous, and it follows readily from (17) and Lemma $5.2\left(\right.$ a) that $z_{m}(\beta, J)$ is decreasing in $J$.

Next, by Theorem 26.3 of ref. 21 , the set $D_{\beta, J}$ is empty (i.e., $G_{\beta, J}^{*}$ is differentiable) if and only if $G_{\beta, J}^{* *}$ is strictly convex, and this holds if and only if $G_{\beta, J}$ is strictly convex. Moreover, Eq. (17), assumption (A1) and Lemma 5.2(a) imply that the second derivative $G_{\beta, J}^{\prime \prime}$ exists everywhere except at the critical point $\rho=D / \beta J$. At this point, we will always consider the rightsided second derivative $G_{\beta, J}^{\prime \prime}(D / \beta J)$ which exists because of Lemma 5.2(e). Also, $G_{\beta, J}^{\prime \prime}$ is piecewise analytic. Its zeroes thus form a countable set. Hence $G_{\beta, J}$ is strictly convex if and only if $G_{\beta, J}^{\prime \prime}(\rho) \geqslant 0$ for all $\rho>0$. This leads us to the following criterion for the existence of a first-order phase transition.

Proposition 5.4. For any $\beta>0$ and $J \geqslant 0$ the following assertions are equivalent.

(a) For at least some $\rho>0$,

$$
G_{\beta, J}^{\prime \prime}(\rho) \equiv \frac{1}{\rho}+\beta g^{\prime \prime}(\rho)-\beta J\left(\varphi \circ h_{*}\right)^{\prime}(\beta J \rho)<0
$$

(b) There exists at least one $z>0$ such that $\# \cdot \mathscr{M}(z, \beta, J)>1$. 
In this case one can choose $z=\exp \left(G_{\beta, J}^{* *}\right)^{\prime}(\rho)$, and this is the unique $z$ for which $\rho$ belongs to the convex hull of $\mathscr{M}(z, \beta, J)$.

We conclude this section with the proof of Lemma 5.2.

Proof of Lemma 5.2. (a) follows from Lemma 5.1(b) and the implicit function theorem. (b) and (d) are obvious because $\varphi^{\prime} \leqslant 1$ and $\varphi^{\prime}(h) \rightarrow 1$ as $h \rightarrow \infty$. For (c), we note that

$$
\begin{aligned}
\int \sigma_{1}^{4} d \lambda & =\int_{0}^{1} s^{4}\left(1-s^{2}\right)^{(D-3) / 2} d s / \int_{0}^{1}\left(1-s^{2}\right)^{(D-3) / 2} d s \\
& =B\left(\frac{5}{2}, \frac{D-1}{2}\right) / B\left(\frac{1}{2}, \frac{D-1}{2}\right)=\frac{3}{D(D+2)}
\end{aligned}
$$

where $B$ stands for the beta function. Hence

$$
\varphi^{i v}(0)=\int \sigma_{1}^{4} d \lambda-3\left(\int \sigma_{1}^{2} d \lambda\right)^{2}=-\frac{6}{D^{2}(D+2)}
$$

Writing $h_{*}(a)=a \varphi^{\prime}\left(h_{*}(a)\right)$ and expanding $\varphi^{\prime}$ up to third order we arrive at (c). (e) follows from (c) by an expansion of $\varphi$; recall from Lemma 5.1 that $\varphi^{\prime \prime}(0)=1 / D$.

(f) Differentiating the relation $h_{*}(a)=a \varphi^{\prime} \circ h_{*}(a)$ we find that $h_{*}^{\prime}(a)$ $=\varphi^{\prime} \circ h_{*}(a) /\left(1-a \varphi^{\prime \prime} \circ h_{*}(a)\right)$ for all $a>D$. In view of $(\mathrm{b}), \varphi^{\prime} \circ h_{*}(a) \rightarrow 1$ and $a \sim h_{*}(a)$ as $a \rightarrow \infty$. Therefore we only need to show that $h \varphi^{\prime \prime}(h) \rightarrow 0$ as $h \rightarrow \infty$. For $D=1$, this is obvious because then $\varphi^{\prime \prime}(h)=(\cosh h)^{-2}$. So let $D>1$. Setting $\delta=h^{-3 / 4}$ and writing $\mathbb{E}_{\mathbf{h}}$ and $V_{\mathbf{h}}$ for the expectation resp. variance relative to $\lambda_{\mathbf{h}}$ with $\mathbf{h}=(h, 0, \ldots, 0)$, we obtain

$$
\begin{aligned}
\varphi^{\prime \prime}(h) & =V_{\mathbf{h}}\left(\sigma_{1}\right) \leqslant \mathbb{E}_{\mathbf{h}}\left(\sigma_{1}-1+\delta\right)^{2} \\
& \leqslant \delta^{2}+\mathbb{E}_{\mathbf{h}}\left(\left(\sigma_{1}-1+\delta\right)^{2} 1_{\left\{\sigma_{1} \leqslant 1-2 \delta\right\}}\right) \\
& \leqslant \delta^{2}+e^{-h \delta} / \lambda\left(\sigma_{1} \geqslant 1-\delta\right) \\
& \leqslant \delta^{2}+e^{-h \delta}(D-1) \delta^{-(D-1) / 2}
\end{aligned}
$$

This shows that $h \varphi^{\prime \prime}(h) \rightarrow 0$ as $h \rightarrow \infty$ and completes the proof of the first statement. The second assertion follows immediately.

(g) Let $D=1, a>1, h=h_{*}(a)$, and $t=\varphi^{\prime}(h)=\tanh h$. From the proof of assertion (f) we know that

$$
\left(\varphi \circ h_{*}\right)^{\prime}(a)=t^{2} h^{\prime}(a)=t^{2} /\left(1-a \varphi^{\prime \prime}(h)\right)=t^{2} /\left(1-a\left(1-t^{2}\right)\right)
$$


Since $h=a t$ by definition of $h$, the stated inequality can be rewritten as $t^{2}<(1+t / h)\left(1-h\left(1-t^{2}\right) / t\right)$. The latter holds if and only if $t^{2}>h^{2}\left(1-t^{2}\right)$, and this is simply the trivial inequality $\sinh ^{2} h>h^{2}$. write

(h) Using the notations and formulas from the proof of (g) we can

$$
1 /\left(\varphi \circ h_{*}\right)^{\prime}(a)=t^{-2}(1-h / t)+h / t
$$

The claimed monotonicity then follows using the series expansion of $h=$ $\operatorname{artanh} t$.

\section{PROOFS}

We introduce the quantity

$$
b(D)=\sup _{a>D}\left(\varphi \circ h_{*}\right)^{\prime}(a)
$$

Lemma $5.2(\mathrm{f})$ ensures that $1 \leqslant b(D)<\infty$. In fact, it follows from Lemma $5.2(\mathrm{e})$, (h) that $b(1)=\left(\varphi \circ h_{*}\right)^{\prime}(1)=3 / 2$, and it is likely that $b(D)=1$ for all $D \geqslant 2$. This would imply that Lemma $5.2(\mathrm{~g})$ holds also for $D>1$, so that the provisos on $D$ in Theorems 3.4 and 3.5 could be avoided. Unfortunately, we could not prove this.

We begin with the proof of Proposition 3.2 on the absence of firstorder phase transitions.

Proof of Proposition 3.2. We use the criterion of Proposition 5.4. For fixed $J$, assumption (A2) implies the existence of some $\rho_{0}>0$ such that $g^{\prime \prime}(\rho) \geqslant J b(D)$ for all $\rho \geqslant \rho_{0}$. Let $\beta$ be so small that $\beta<D / J \rho_{0}$ and $\min _{\rho \leqslant \rho_{0}} g^{\prime \prime}(\rho) \geqslant-1 / \beta \rho_{0}$. Then it is easily seen that there is no $\rho$ satisfying (18). Similarly, if $\beta$ is fixed and $g^{\prime \prime} \geqslant 0$ we let $\rho_{0}$ be so large that $g^{\prime \prime}(\rho) \geqslant b(D)$ for all $\rho \geqslant \rho_{0}$ and $J \leqslant 1$ so small that $J \leqslant D / \beta \rho_{0}$. The result then follows as in the first case.

Next, we apply Proposition 5.4 to establish the existence of a firstorder transition from nonmagnetic vapor to ferromagnetic liquid, as stated in Theorem 3.3. Since $\rho=D / \beta J$ is the critical density for the incipience of ferromagnetic order, we only need to insert this critical value into (18).

Proof of Theorem 3.3. By Lemma 5.2(e), the right-sided derivative of $\varphi \circ h_{*}(a)$ at the critical value $a=D$ exists and is equal to $(D+2) / 2 D$. Hence, $G_{\beta, J}^{\prime \prime}(D / \beta J)<0$ if and only if the hypothesis of the theorem holds. The result thus follows from Proposition 5.4. 
Postponing the proofs of Theorems 3.4 and 3.5 until the end of this section, we now turn to the proof of Corollary 3.6 on the first-order transition between magnetic phases.

Proof of Corollary 3.6. Let $b(D)$ be as in (19). We fix some $\left.\rho_{0} \in\right] 0, \rho_{\min }[$ and let $J>0$ be so small that

(i) $\min _{\rho \leqslant p_{0}} g^{\prime \prime}(\rho) \geqslant J b(D)$;

(ii) the set $\left\{g^{\prime \prime}<J\right\}$ is an interval $] \rho_{1}, \rho_{2}\left[\right.$ satisfying $\rho_{0}<\rho_{1}<\rho_{\text {min }}$ $<\rho_{2}$, so that $g^{\prime}(\rho)-J \rho$ has a local maximum at $\rho_{1}$ and a local minimum at $\rho_{2}$ and is strictly monotone on the intervals which are separated by these points; and

(iii) $g^{\prime}\left(\rho_{0}\right)-J \rho_{0}<g^{\prime}\left(\rho_{2}\right)-J \rho_{2}$.

(i) is possible because $g^{\prime \prime}$ is positive on the left of $\rho_{0}$, (ii) uses the Taylor expansion of $g^{\prime \prime}$ at $\rho_{\min }$, and (iii) merely says that the interval $] \rho_{1}, \rho_{2}[$ on which $g^{\prime}(\rho)-J \rho$ decreases is short enough compared with the interval ]$\rho_{0}, \rho_{1}[$ of increase. Recalling that the construction of the convex envelope corresponds to Maxwell's equal-area construction for the derivative, we see that our conditions imply that the convex envelope of $g(\rho)-J \rho^{2} / 2$ has a unique amine piece on an interval $\left[r_{-}, r_{+}\right]$, where $r_{-}>0$ due to (iii). Hence, if $\beta$ is large enough then $D / \beta J<\rho_{--}$for the $\rho_{-}$in Theorem 3.4(a), so that the corollary follows from this theorem.

The next result to prove is Proposition 3.7 on first-order transitions in the nonmagnetic regime.

Proof of Proposition 3.7. Our assumptions on $g$ and $\beta$ imply that there is a rightmost interval $I$ on which $G_{\beta, 0}^{\prime \prime}$ is negative. By Proposition $4.3, I$ is contained in some maximal interval $\left[\rho_{-}, \rho_{+}\right]$on which $G_{\beta, 0}^{* *}$ is affine. Let $\ln z$ be the corresponding slope.

Next, assumption (A2) gives us some $\rho_{1}>\rho_{+}$such that $g^{\prime \prime} \geqslant b(D)$ on $\left[\rho_{1}, \infty[\right.$. Let $\left.J \in] 0,1\right]$ be so small that $D / \beta J>\rho_{1}$. Looking at (18) we then see that $G_{\beta, J}^{\prime \prime} \geqslant 0$ on $\left[\rho_{1}, \infty[\right.$. On the other hand, we also have $G_{\beta, J}^{\prime \prime}=G_{\beta, 0}^{\prime \prime} \geqslant 0$ on $\left[\rho_{+}, D / \beta J\right]$. Consequently, $G_{\beta, J}$ is convex on $\left[\rho_{+}, \infty[\right.$ and coincides with $G_{\beta, 0}$ on (a neighborhood of) $\left[0, \rho_{+}\right]$. This shows that $\left[\rho_{-}, \rho_{+}\right]$is also the rightmost maximal interval on which $G_{\beta, J}^{* *}$ is affine, and the proposition follows.

We proceed with the proof of Theorem 3.8 on the existence of triple points.

Proof of Theorem 3.8. Step 1: Existence of $z_{c}$. Let $\beta>\beta_{\min }$ be so close to $\beta_{\min }$ that $\left\{\rho>0: \rho g^{\prime \prime}(\rho)<-1 / \beta\right\}$ is an interval. This is possible 
because, by analyticity, $\rho g(\rho)$ is convex in a neighborhood of $\rho_{\min }$. Then $\left\{G_{\beta, 0}^{\prime \prime}<0\right\}$ is an interval containing $\rho_{\min }$. Hence there is a unique interval $\left[\rho_{\ldots}, \rho_{\sharp}\right] \ni \rho_{\min }$ (depending on $\beta$ ) on which $G_{\beta, 0}^{* *}$ is affine. Let $z_{c}=z_{c}(\beta)>0$ be such that $\ln z_{c}$ is the corresponding slope.

We observe that $\rho_{\#}=\rho_{\#}(\beta) \rightarrow \rho_{\min }$ as $\beta \rightarrow \beta_{\min }$. Indeed, otherwise there would exist some $\varepsilon>0$ and a sequence $\beta_{n} \rightarrow \beta_{\min }$ such that $\rho_{\#}\left(\beta_{n}\right)-\rho_{-}\left(\beta_{n}\right) \geqslant \varepsilon$ for all $n$. By assumption (A2), the sequence $\left(\rho_{\#}\left(\beta_{n}\right)\right)$ is bounded. Considering suitable limit points and using that $G_{\beta_{n}, 0}^{\prime}$ converges to $G_{\beta_{\min }, 0}^{\prime}$ locally uniformly we then see that $G_{\beta_{\min }, 0}^{\prime}$ would take the same value at two points of distance $\varepsilon$. This is impossible because $G_{\beta_{\min }, 0}^{\prime}$ is strictly increasing by assumption on $g$.

It follows from the preceding observation that $\beta \rho_{\#} g^{\prime \prime}\left(\rho_{\#}\right) \rightarrow-1$ as $\beta \rightarrow \beta_{\min }$. Consequently, choosing $\beta$ close enough to $\beta_{\min }$ we can achieve that $\beta \rho_{\sharp} g^{\prime \prime}\left(\rho_{\sharp}\right)<D / 2$. In the following, we will consider a fixed such $\beta$. We then can assume for simplicity that $\ln z_{c}=0$ and $G_{\beta, 0}\left(\rho_{-}\right)=G_{\beta, 0}\left(\rho_{\#}\right)=0$. (Otherwise we add a linear term to $g$.)

Step 2: Existence of $J_{c}$. Let $J_{\#}>0$ be defined by the equation $\rho_{\#}=D / \beta J_{\#}$. By Lemma 5.2(e) and (18), it then follows from Step 1 that

$$
G_{\beta, J_{\sharp}}^{\prime \prime}\left(\rho_{\#}\right)=\frac{1}{\rho_{\#}}+\beta g^{\prime \prime}\left(\rho_{\#}\right)-\frac{D}{\rho_{\#}} \frac{D+2}{2 D}<0
$$

By the final assumptions of Step 1 , this implies that $q\left(J_{\#}\right)<0$, where

$$
q(J)=\inf _{\rho>D / \beta J} G_{\beta, J}(\rho)
$$

By Lemma 5.2(a) and (17), $q(J)$ is strictly decreasing on $\left[0, J_{\#}\right]$. Using (A2) and the locally uniform convergence of $G_{\beta, J_{n}}$ to $G_{\beta, J}$ when $J_{n} \rightarrow J$, we also see that $q(J)$ is continuous. Finally, we know from the proof of Proposition 3.7 that $q(J)>0$ if $J$ is small enough. Consequently, there is a unique $\left.J_{c}=J_{c}(\beta) \in\right] 0, J_{\#[}$ such that $q\left(J_{c}\right)=0$. On $\left[\rho_{\sharp}, D / \beta J_{c}\left[, G_{\beta, J_{c}}\right.\right.$ equals $G_{\beta, 0}$, which is strictly convex and increasing on this interval. So, the infimum defining $q\left(J_{c}\right)=0$ is attained for at least one $\rho_{+}>D / \beta J_{c}$. This proves the first assertion of the theorem.

Step 3: \# $\mathscr{M}(z, \beta, J)=3$. Suppose that $D=1$ and $g^{\prime \prime}$ is convex. Then $G_{\beta, 0}^{\prime \prime}$ is convex (cf. (18)) and thereby strictly increasing on $\left[\rho_{\#}, \infty\right.$ [. Using Lemma $5.2(\mathrm{~h})$, we conclude that $G_{\beta, J_{c}}^{\prime \prime}$ is strictly increasing on $\left[1 / \beta J_{c}, \infty[\right.$, and therefore strictly positive on $\left[\rho_{+}, \infty\left[\right.\right.$, where $\rho_{+}$is the smallest number $>1 / \beta J_{c}$ satisfying $G_{\beta, J_{c}}\left(\rho_{+}\right)=q\left(J_{c}\right)=0$. Hence $G_{\beta, J_{c}}$ is strictly convex on $\left[\rho_{+}, \infty\left[\right.\right.$, so that there cannot exist any other $\rho>\rho_{+}$with 
$G_{\beta, J_{c}}(\rho)=q\left(J_{c}\right)=0$. This shows that $\mathscr{M}\left(z, \beta, J_{c}\right)=\left\{\rho_{-}, \rho_{\sharp}, \rho_{+}\right\}$. In the next two steps, we assume that the last identity holds.

Step 4: The case $J>J_{c}$. Let $\left.\left.J \in\right] J_{c}, J_{\#}\right]$. Then $q(J)<0$, but $G_{\beta, J}=G_{\beta, 0} \geqslant 0$ on $\left.] 0, D / \beta J\right]$. Hence $G_{\beta, J}^{* *}$ is affine at least on $\left[\rho_{-}, D / \beta J\right]$ with negative slope $\ln z_{J} \equiv \ln z_{m}(\beta J)$. Let $\left[\rho_{J,-}, \rho_{J,+}\right] \supset\left[\rho_{-}, D / \beta J\right]$ be the maximal interval on which $G_{\beta, J}^{* *}$ has slope $\ln z_{J}$. Then $\rho_{J, \ldots}, \rho_{J,+} \in$ $\mathscr{M}\left(z_{J}, \beta, J\right)$. In the limit as $J \downarrow J_{c}$, all accumulation points of $\left(\rho_{J_{-}-}\right)$and $\left(\rho_{J,+}\right)$ belong to $\mathscr{M}\left(z_{c}, \beta, J_{c}\right)$, by Theorem 3.1(iii). Since $\rho_{J,-}<\rho_{-}$and $\rho_{J,+} \geqslant D / \beta J$, it follows that $\rho_{J,-} \rightarrow \rho_{-}$and $\rho_{J_{+}+} \rightarrow \rho_{+}$. Assertion (a) is thus proved.

Step 5: The case $J<J_{c}$. From Step 2 we know that $G_{\beta, J_{c}}\left(D / \beta J_{c}\right)>0$ $=q\left(J_{c}\right)=G_{\beta, J_{c}}\left(\rho_{+}\right)$. Let $J<J_{c}$ be so close to $J_{c}$ that still $G_{\beta, J}(D / \beta J)>$ $q(J)$. We then see that $G_{\beta, J}(D / \beta J)>G_{\beta, J}^{* *}(D / \beta J)$, whence $G_{\beta, J}^{* *}$ has slope $\ln z_{J} \equiv \ln z_{m}(\beta, J)$ on an interval $\left[\rho_{J,-}, \rho_{J,+}\right]$ with $\rho_{\#}<\rho_{J,-}<D / \beta J<$ $\rho_{J,+}$. (The first inequality holds because $q(J)>0$ ). As $J \uparrow J_{c}$,

$$
G_{\beta, 0}^{\prime}\left(\rho_{J,-}\right)=\ln z_{J} \downarrow \ln z_{c}=0=G_{\beta, 0}^{\prime}\left(\rho_{\#}\right)
$$

and therefore $\rho_{J,-} \downarrow \rho_{\#}$. Arguing as in Step 4 we also see that $\rho_{J,+} \rightarrow \rho_{+}$. The rest of statement $(b)$ is evident.

Finally we provide the proof of Theorem 3.4 on the low temperature limit. The proof of Theorem 3.5 is similar but simpler and will be omitted.

Proof of Theorem 3.4. Let $J \geqslant 0$ be given and $\left[r_{-}, r_{+}\right]$be any critical interval of $g_{J}$. We can assume that $\gamma=0$ and $g_{J}^{* *}=0$ on $\left[r_{-}, r_{+}\right]$. (Otherwise we replace $g$ by $\tilde{g}(\rho)=g(\rho)-\gamma \rho-c$ for suitable $c \in \mathbf{R}$.) We consider the scaled functions $g_{\beta, J}=\beta^{-1} G_{\beta, J}$. For any $\rho>0$ we have

$$
g_{\beta, J}^{\prime}(\rho)=g_{J}^{\prime}(\rho)+\beta^{-1} \ln \rho+\left[J \rho-\beta^{-1} \varphi \circ h_{*}(\beta J \rho)\right]
$$

and

$$
g_{\beta, J}^{\prime \prime}(\rho)=g_{J}^{\prime \prime}(\rho)+\frac{1}{\beta p}+J\left[1-\left(\varphi \circ h_{*}\right)^{\prime}(\beta J \rho)\right]
$$

Step 1: Choice of $\delta$. Let $\varepsilon>0$ be given. We can assume that $\varepsilon$ is so small that $g_{J}^{* *}$ is strictly convex on $\left[r_{+}, r_{+}+\varepsilon\right]$ and, if $r_{-}>0$, also on $\left[r_{-}-\varepsilon, r_{-}\right]$. We may also require that $L \equiv r_{+}-r_{-}-2 \varepsilon>0$ and, if $r_{-}>0$, also $r_{-}-\varepsilon>0$. Since $g_{J}>g_{J}^{* *}=0$ on $] r_{-}, r_{+}[$, there exists some $\delta>0$ such that $g_{J}>3 \delta$ on $\left[r_{-}+\varepsilon, r_{+}-\varepsilon\right]$. We also require that $\delta$ is so small that $g_{J}(\rho) \geqslant 2 \delta+\left(\rho-r_{+}\right) 2 \delta / L$ for all $\rho \geqslant r_{+}+\varepsilon$. This is possible because 
$g_{J}^{* *}$ is strictly convex on $\left[r_{+}, r_{+}+\varepsilon\right]$. Similarly, if $r_{-}>0$ we also stipulate that $g_{J}(\rho) \geqslant 2 \delta-\left(\rho-r_{-}\right) 2 \delta / L$ for all $\rho \leqslant r_{-}-\varepsilon$. Finally, we can assume that $2 \delta / L<\varepsilon$.

Step 2: Condition on $\beta$. Recall that

$$
\begin{aligned}
& g_{\beta, J}(\rho)-g_{J}(\rho) \\
& =[\rho \ln \rho-\rho] / \beta+\frac{J \rho^{2}}{2}+\rho \frac{h_{*}(\beta J \rho)}{2 \beta} \varphi^{\prime} \circ h_{*}(\beta J \rho)-\rho \frac{\varphi \circ h_{*}(\beta J \rho)}{\beta}
\end{aligned}
$$

By the bounds in Lemma 5.2(b),(d), there exists some $\eta_{1}>0$ such that, for all $\beta \geqslant 1,\left|g_{\beta, J}-g_{J}\right|<\delta$ on $\left[0, \eta_{1}\right]$. By assumption (A2), there exists some $\eta_{2}>1$ such that $g_{J}^{\prime}>2 \delta / L$ on $\left[\eta_{2}, \infty[\right.$. (Eq. (20) and again the bound in Lemma $5.2(\mathrm{~d})$ then show that, for all $\beta, g_{\beta, J}^{\prime}>2 \delta / L>0$ on $\left[\eta_{2}, \infty[\right.$.) We can assume that $\eta_{2}>r_{+}+\varepsilon$. In view of the asymptotics in Lemma $5.2(\mathrm{~b})(\mathrm{d})$, we now can choose $\beta$ so large that $\left|g_{\beta, J}-g_{J}\right|<\delta$ on $\left[\eta_{1}, \eta_{2}\right]$.

Step 3: Proof of assertion (a). Let $\beta$ satisfy the requirement of Step 2. Then, on the interval $\left[r_{-}+\varepsilon, r_{+}-\varepsilon\right]$, the inequality $g_{\beta, J}>g_{J}-\delta>2 \delta$ holds. On the other hand, since $g_{\beta, J}^{* *}$ is convex and $g_{\beta, J}^{* *}\left(\rho_{+1-}^{*}\right) \leqslant$ $g_{\beta, J}\left(\rho_{+/-}\right)<\delta$, we have $g_{\beta, J}^{* *}<\delta$ on $\left[r_{-}, r_{+}\right]$. Hence $g_{\beta, J}>g_{\beta, J}^{* *}+\delta$ on $\left[r_{-}+\varepsilon, r_{+}-\varepsilon\right]$. This shows that $g_{\beta, J}^{* *}$ is affine at least on this interval. Let $q=q_{\beta, J}$ be the associated slope and $\left[\rho_{-}, \rho_{+}\right] \supset\left[r_{-}+\varepsilon, r_{+}-\varepsilon\right]$ be the maximal interval on which $g_{\beta, J}^{* *}$ is affine with this slope. (By assumption (A2), $\rho_{+}<\infty$.) It is then evident that, for $z=z_{\beta, J}=\exp \beta q, \rho_{-}, \rho_{+} \epsilon$ $\mathscr{M}(z, \beta, J)$.

To estimate $q$ and $\rho_{-1+}$ we note that $g_{\beta, J}>-\delta$ on $\left[0, \eta_{2}\right]$ and $g_{\beta, J}^{\prime}>0$ on $\left[\eta_{2}, \infty\left[\right.\right.$. Hence $g_{\beta, J}>-\delta$ everywhere and therefore $g_{\beta, J}^{* *}>-\delta$. This gives us the estimate

$$
|q|=\left|g_{\beta, J}^{* *}\left(r_{+}-\varepsilon\right)-g_{\beta, J}^{* *}\left(r_{-}+\varepsilon\right)\right| / L<2 \delta / L<\varepsilon
$$

This in turn implies that for all $p \in\left[r_{+}+\varepsilon, \eta_{2}\right]$

$$
\begin{aligned}
g_{\beta, J}(\rho) & >g_{J}(\rho)-\delta>\delta+\left(\rho-r_{+}\right) 2 \delta / L \\
& >g_{\beta, J}^{* *}\left(r_{+}\right)+\left(\rho-r_{+}\right) q
\end{aligned}
$$

Hence $\rho_{+} \notin\left[r_{+}+\varepsilon, \eta_{2}\right]$. By our assumption on $\eta_{2}$, the case $\rho_{+}>\eta_{2}$ is also impossible. Hence $\rho_{+}<r_{+}+\varepsilon$. In the case $r_{-}>0$, the same reasoning shows that $\rho_{-}>r_{-}-\varepsilon$. This completes the proof of $(a)$.

Step 4: Proof of $(b)$. We suppose first that $r_{-}>0$. In addition to the conditions in Step 1, we assume that $\varepsilon$ is so small that $g_{J}^{\prime \prime}>0$ on 
$\left[r_{-}-\varepsilon, r_{-}+\varepsilon\right] \cup\left[r_{+}-\varepsilon, r_{+}+\varepsilon\right]$, and then require that $\delta$ is also such that $g_{J}^{\prime \prime}>\delta$ on the same set. Lemma 5.2(f) implies that $\left|g_{\beta, J}^{\prime \prime}-g_{J}^{\prime \prime}\right|<\delta$ on $\left[r_{-}-\varepsilon, \infty\right.$ [ when $\beta$ is large enough. For these $\beta, g_{\beta, J}$ is strictly convex on the intervals $\left[r_{-}-\varepsilon, r_{-}+\varepsilon\right]$ and $\left[r_{+}-\varepsilon, r_{+}+\varepsilon\right]$ containing $\rho_{-}$resp. $\rho_{+}$, whence $g_{\beta, J}>g_{\beta, J}^{* *}$ on $] \rho_{-}, \rho_{+}[$. This proves assertion (b) in the case $r_{-}>0$.

If $r_{-}=0$, the conditions on $\varepsilon$ and $\delta$ involving $g_{J}^{\prime \prime}$ in the neighborhood of $r_{-}$are replaced by the condition that $g_{J}^{\prime}>\delta+2 \delta / L$ on $[0, \varepsilon]$. Let $b(D)$ be as in (19) and $b<\infty$ be such that $g^{\prime \prime}-J b(D)>-b$ on $[0, \varepsilon]$. By (21), it then follows that, for any $\beta>1 / \varepsilon b, g_{\beta, J}^{\prime \prime}>0$ on $[0,1 / \beta b]$. Let $\beta$ be so large that, in addition to our previous assumptions, $\beta^{-1} \ln (\beta b)<\delta$. Equation (20) and Lemma 5.2(d) then show that $g_{\beta, J}^{\prime}>g_{J}^{\prime}-\delta>2 \delta / L>q$ on $[1 / \beta b, \varepsilon]$. This implies that $\rho_{-} \notin[1 / \beta b, \varepsilon]$. It follows that $g_{\beta, J}$ is strictly convex on a neighborhood of $\rho_{-}$, and thus $g_{\beta, J}>g_{\beta, J}^{* *}$ on $] p_{-}, \rho_{+}[$also in the case $r_{-}=0$.

Step 5: Proof of $(\mathrm{c})$. By Proposition 5.4, the uniqueness of $z$ is equivalent to the fact that $g_{\beta, J}$ is strictly convex on the intervals $\left[0, \rho_{-}\right]$and $\left[\rho_{+}, \infty\left[\right.\right.$. If $D=1$, Lemma $5.2(\mathrm{~g})$ and (21) show that $g_{\beta, J}^{\prime \prime}>g_{J}^{\prime \prime}$ everywhere. So, in case $D=1$ the assertion follows immediately from our assumptions. In the alternate case we argue as follows. For $\varepsilon$ as in Step 4, it follows from (A2) that $g_{J}^{\prime \prime}>\delta$ on $\left[r_{+}-\varepsilon, \infty[\right.$ for some $\delta>0$. From Lemma 5.2(f) and (21) we then see that $g_{\beta, J}^{\prime \prime}>0$ on this interval when $\beta$ is large enough. If $r_{-}>0$, we can further assume that $g_{J}^{\prime \prime}>\delta$ on $\left[0, r_{-}+\varepsilon\right]$. Again by Lemma 5.2(f), we can find some $\alpha>0$ such that $1-\left(\varphi \circ h_{*}\right)^{\prime}(\beta J \rho)>-\delta / J$ for all $\rho \geqslant \alpha / \beta$. In view of (21), this shows that $g_{\beta, J}^{\prime \prime}>0$ on $\left[\alpha / \beta, r_{-}+\varepsilon\right]$. But our additional assumption for the case $D>1$ implies that $g_{\beta, J}^{\prime \prime}>0$ also on $[0, \alpha / \beta]$ when $\beta$ is sufficiently large. This completes the proof of assertion $(\mathrm{c})$.

\section{PHYSICAL COMMENTS}

Although the phenomenon of ferromagnetism is usually associated with matter in the solid state, there is an indication that it also occurs in liquid ferromagnetic materials such as the AuCo and CoPd alloys, cf. refs. 18 and 28. This led to intensive theoretical investigations (including refs. $11,13,18,24,26,27$, and 31 ) and is the main physical justification of the present work.

As we have stated in the introduction, a main feature of "soft" magnetic materials is an interplay of magnetic and molecular forces. For magnets on soft lattices, this phenomenon is known as magnetostriction 
and leads to a first-order phase transition with simultaneous jumps of magnetization and density; cf., e.g., ref. 19 and the references therein. Close to that, an annealed site-diluted lattice model of agglomeration of ferromagnetis particles has been considered (by a mean-field approximation) in ref. 24 , while a rigorous study of such class of models is rather recent, see refs. 4 and 30 .

The rich variety of phase transitions in our model with Hamiltonian (2) is also similar to the behavior of another kind of "soft" material, namely liquid crystals. In fact it is known (see e.g., ref. 20) that a transition between the so-called $\mathrm{C}$-A smectic phases can be continuous while the next transition from the smectic $\mathrm{A}$ to the nematic phase $\mathrm{N}$ is usually discontinuous with a jump of density, cf. our scenario B. The standard explanation of this phenomenon ${ }^{(20)}$ is based on an interplay of smectic density order and the magnitude of the nematic alignment: this corresponds to positional resp. orientational order in our model. Our scenario A can be realized in the case of the order-disorder transition in nematics when the third order term in the Landau expansion of the free energy is absent. In this case it is known that there is a "weak" first-order phase transition to an ordered phase together with a jump of density, which is again due to the interplay of positional and orientational order; cf. refs. 20 or 7.

The interplay of magnetic and molecular forces also explains transitions in a new kind of magnetic and dipolar fluids, the ferrocolloids etc, which are intensively studied, see e.g., refs. 11, 24, 26, 27, and 28. These systems are stable suspensions of dispersed ferroparticles in liquids (ferrofluid emulsions). An effective attraction between particles due to magnetic moments leads to a variety of phenomena such as nucleation into magnetic dropletlike aggregates, phase separation induced by magnetic fields, and even solidification into chains -which is a first-order phase transition; cf. refs. 26, 32, and 33 and the references there. To study these phenomena we would need to modify our model in such a way that it provides a local description of the interplay of forces. This means that the mean-field interaction of our model should be replaced by a suitable shortrange interaction as in refs. 9 and 12 .

In refs. 11,27 , and 28 , the phase diagram and the spatial structure of Heisenberg and dipolar fluids were studied by numeric simulation and a modified mean-field theory in the presence and the absence of the external field. The phase diagrams obtained there are comparable to our scenario A.

In conclusion we would like to mention that the interplay of forces studied in the present paper has also been observed in the case of quantum degrees of freedom. ${ }^{(2,25)}$ Theoretical studies and computer simulations of classical fluids with internal quantum states show that additional attractive pair interactions are "turned on" when the internal states are hybritized. 
The resulting phase diagram corresponds to our scenario $\mathrm{A}$, while there is a specific quantum regime when there is no transition for any densities. ${ }^{(25)}$

\section{ACKNOWLEDGMENTS}

H.O.G. gratefully acknowledges warm hospitality at the Centre de Physique Théorique in Marseille-Luminy and V.Z. at the Mathematical Institute of the University of Munich. We thank Joel Lebowitz for attracting our attention to the interplay in the case of quantum internal states. We also thank an anonymous referee for some recent references concerning physical results in this actively developping area.

\section{REFERENCES}

1. N. Angelescu and V. A. Zagrebnov, A generalized quasiaverage approach to the description of the limit states of the $n$-vector Curie-Weiss ferromagnet, J. Stat. Phys. 41:323-334 (1985).

2. P. Ballone, Ph. de Smedt, J. L. Lebowitz, J. Talbot, and E. Waisman, Computer simulation of a classical fluid with internal quantum states, Phys. Rev. A 35:942-944 (1987).

3. T. Burke, J. L. Lebowitz, and E. Lieb, Phase transition in a model quantum system: Quantum corrections to the location of the critical point, Phys. Rev. 149:118-122 (1966).

4. L. Chayes, S. B. Shlosman, and V. A. Zagrebnov, Discontinuity of the magnetization in diluted O(n)-model, Preprint (CPT, Luminy, Marseille, 1998).

5. I. Csiszár, Sanov property, generalized I-projection and a conditional limit theorem, Ann. Prob. 12:768-798 (1984).

6. D. J. Gates and O. Penrose, The van der Waals limit for classical systems. I: A variational principle, Commun. Math. Phys. 15:255-276 (1969).

7. P. G. de Gennes. The Physics of Liquid Crystals (Oxford University Press, Oxford, 1974).

8. H. O. Georgii, Gibhs Measures and Phase Transitions, De Gruyter Studies in Mathematics, Vol. 9 (de Gruyter, Berlin, 1988).

9. H. O. Georgii and O. Häggström, Phase transition in continuum Potts models, Commun. Math. Phys. 181:507-528 (1996).

10. H. O. Georgii and H. Zessin, Large deviations and the maximum entropy principle for marked point random fields, Prohab. Theory Relat. Fields 96:177-204 (1993).

11. B. Groh and S. Dietrich, Spatial structure of dipolar ferromagnetic liquids, Phys. Rev. Lett. 79:749-752 (1997).

12. Ch. Gruber and R. B. Griffiths, Phase transition in a ferromagnetic fluid, Physica $A$ 138:220-230 (1986).

13. P. C. Hemmer and D. Imbro, Ferromagnetic fluids, Phys. Rev. A 16:380-386 (1977).

14. K. Johansson, On separation of phases in one-dimensional gases, Commun. Math. Phys. 169:521-561 (1995).

15. J. L. Lebowitz and O. Penrose, Rigorous treatment of the Van der Waals Maxwell theory of the liquid vapour transition, J. Math. Phys. 7:98-113 (1966).

16. J. L. Lebowitz, A. E. Mazel, and E. Presutti, Liquid-vapor phase transitions for systems with finite range interactions, J. Stat. Phys. (to appear, 1998).

17. E. Lieb, Quantum-mechanical extension of the Lebowitz-Penrose theorem on the Van der Waals theory, J. Math. Phys. 7:1016-1024 (1966). 
18. E. Lomba, J.-J. Weis, N. G. Almarza, F. Bresme, and G. Stell, Phase transitions in a continuum model of the classical Heisenberg magnet: The ferromagnetic system, Phys. Rer. E 49:5169-5178 (1994).

19. D. C. Mattis, The Theory of Magnetism (Harper \& Row, New York, 1965).

20. S. A. Pikin, Structural Transformations in Liquid Crystals (in Russian) (Moscow, Nauka, 1981 ).

21. R. T. Rockafellar, Concex Analysis (Princeton University Press, Princeton, N.J., 1970).

22. D. Ruelle, Existence of a phase transition in a continuous classical system, Phys. Ret. Lett. 27:1040-1041 (1971).

23. B. Simon, The Statistical Mechanic: of Latice Gases, Vol. I (Princeton University Press, Princeton, N.J., 1993).

24. K. Sano and M. Doi. Theory of agglomeration of ferromagnetic particles in magnetic fluids, J. Phys. Soc Japan 52:2810-2815 (1983).

25. Ph. de Smedt, P. Nielaba, J. L. Lebowitz, J. Talbot, and L. Dooms, Static and dynamic correlations in fluids with internal quantum states: Computer simulations and theory, Phys. Ret. A 38:1381-1394 (1988).

26. M. J. Stevens and G. S. Grest, Structure of soft-sphere dipolar fluids, Phys. Rev. E 51:5962-5975 (1995).

27. M. J. Stevens and G. S. Grest, Phase coexistence of a Stockmayer fluid in an applied field. Phys. Rer. E 51:5976-5983 (1995).

28. J.-J. Weis, M. J. P. Nijmeijer, J. M. Taveres, and M. M. Telo de Gama, Phase diagram of Sleisenberg fluids: Computer simulation and density functional theory, Phys. Rev. E 51:5962-5975 (1997).

29. B. Widom and J. S. Rowlinson, New model for the study of liquid-vapor phase transition, J. Chem. Phys. 52:1670-1684 (1970).

30. V. A. Zagrebnov, Long-range order in a lattice-gas model of nematic liquid crystals, Physica A 232:737-746 (1996).

31. H. Zhang and M. Widom, Global phase diagrams for dipolar lluids, Phys. Ree. E 49:R359l-R3593 (1994).

32. Zhu Yun, E. Haddadian, T. Mou, M. Gross, and Jing Liu, Rôle of nucleation in the structure evolution of a magnetorheological fluid, Phys. Ret. E 53:1753-1759 (1996).

33. A. Yu. Zubarev and A. O. Ivanov, Kinetics of a magnetic fluid phase separation induced by an external magnetic field, Phys. Rer. E 55:7192-7202 (1997). 